\title{
Especificação de Perfis de Grupos para Suporte à Formação de Grupos Colaborativos na EAD
}

\author{
Felipe O. M. Cunha ${ }^{1}$, Clauirton de Albuquerque Siebra ${ }^{1}$ \\ ${ }^{1}$ Centro de Informática - Universidade Federal da Paraíba (UFPB) \\ João Pessoa - PB - Brasil \\ felipe.cunha@dce.ufp.b.br; cas@cin.ufpe.br
}

\begin{abstract}
This work discusses the formation of learning groups in the context of distance education. As from a review study in literature, it was acknowledged that the existing approaches did not take in consideration the tutor's criteria and preferences in a distance education program, what may bring about the failure of the whole group working. Based on a survey of tutors, it was achieved the specification of groups' profiles in order to contemplate tutors' criteria and preferences.
\end{abstract}

Resumo. Este trabalho discute a formação de grupos de aprendizagem no contexto da Educação a Distância. A partir de estudo de revisão na literatura, verificou-se que as abordagens existentes não consideram as preferências de tutores a distância, o que pode acarretar no fracasso do trabalho em grupo. Com base em pesquisa realizada com tutores, foi feita a especificação de perfis de grupos considerando critérios e preferências de tutores.

\section{Introdução}

Há uma grande preocupação com a formação acadêmica a distância. Pesquisas revelam que o desempenho dos estudantes está diretamente relacionado aos objetivos de aprendizagem, às atividades e às estratégias de ensino [Prado, 2006], [Ramos et al., 2014]. O desempenho insatisfatório dos alunos remete à necessidade de considerar aspectos relacionados a como eles se organizam colaborativamente diante de situações de aprendizagem [Santos et al., 2007]. Conceber estratégia efetiva para atribuir estudantes em grupo não é uma tarefa fácil. Diante disso, discutir a formação de grupos de aprendizagem na $\mathrm{EaD}$ é uma atividade bem vinda e urgente.

Recentemente, muitas iniciativas têm acreditado no potencial da Aprendizagem Colaborativa Apoiada por Computador, do inglês Computer Supported Collaborative Learning - CSCL, como instrumento de aumento do poder cognitivo e da produtividade na academia [Brito et al., 2011]. A CSCL preocupa-se em como os estudantes utilizam sistemas computacionais para trabalhar em grupo e como trabalham conjuntamente para resolver uma tarefa. $\mathrm{O}$ trabalho em grupo é caracterizado pela divisão de um problema complexo em subproblemas e seus membros negociam e compartilham entendimentos relevantes à resolução do problema [Roschelle e Teasley, 1995], [Dillenbourg, 1999].

Pesquisas enfatizam a necessidade de que os estudantes partam do aprendizado individual para o trabalho em grupo [Ikeda et al., 1997]. Trabalhar colaborativamente para resolver problemas pode ter inúmeras vantagens. Primeiro, através do ensino recíproco [Palincsar e Brown, 1984], [Rosenshine e Meister, 1994], a troca de conhecimento é estabelecida mais fortemente; alunos mais experientes podem apoiar 


\section{CBIE-LACLO 2015}

Anais dos Workshops do IV Congresso Brasileiro de Informática na Educação (CBIE 2015)

aos demais. Desse modo, acredita-se no trabalho em grupo para, efetivamente, potencializar o aprendizado e elevar a qualidade do processo educativo na $\mathrm{EaD}$.

Diversas pesquisas propõem abordagens para a tarefa de seleção dos membros. Há sugestões de que essa tarefa deva ser compartilhada entre o professor e os alunos [Mahenthiran e Rouse, 2000]. Outras reforçam a ideia de que a formação auto selecionada pode ter melhores resultados que a aleatória [Chapman et al., 2006]. Estabelecer critérios e preferências constitui um passo importante para a formação de grupos. No entanto, embora exista uma vasta quantidade de alternativas para a formação de grupos, ainda não se conseguiu superar de forma sistematizada o problema de atribuir estudantes em grupo. Além disso, não foram encontradas na literatura abordagens que consideram as preferências de tutores para apoiar a tarefa de formação de grupos. Diante disso, este trabalho objetiva especificar perfis de grupos de aprendizagem para apoiar as estratégias de formação, com base nos critérios e nas preferências de tutores a distância.

\section{Trabalhos relacionados}

Os estudos acerca da formação de grupos ainda são incipientes. Entretanto a revisão bibliográfica mostrou que há trabalhos correlatos acerca dessa temática. O estudo de Mahenthiran e Rouse [2000] mostrou que o desempenho e o nível de satisfação do aluno estão diretamente relacionados à forma de seleção dos membros. Seu experimento demonstrou que grupos formados por indicação dos próprios estudantes apresentaram melhores resultados que grupos aleatórios. $\mathrm{O}$ estudo sugere que a tarefa de seleção dos membros deva ser compartilhada entre o professor e os alunos. Já o estudo de Oakley et al., [2004] descreve que, para criar grupos efetivos, é preciso que a tarefa de seleção dos membros seja de responsabilidade do professor. Chapman et. al., [2006] afirmam que a composição aleatória de grupos pode não produzir resultados pedagógicos tão significativos em relação à formação auto selecionada, após verificarem que os estudantes em grupos auto selecionados foram classificados com índices mais elevados sob diversos aspectos importantes para o trabalho em grupo. Entretanto, perceberam maiores índices de conflito entre os membros, sugerindo, portanto, o acompanhamento do professor.

Diante disso, percebe-se que a comunidade científica já sinaliza uma preocupação no sentido de se ter o controle sobre a formação de grupos de estudantes. É preciso extrair as informações caracterizadoras dos grupos, de modo a viabilizar o seu bom desempenho, como retirar as informações do perfil dos estudantes, conforme sugere estudo de Muehlenbrock [2006]. Algumas abordagens tendem a formar grupos maximizando as diferenças entre as características dos estudantes, objetivando formar grupos heterogêneos a partir da mensuração dos níveis de habilidades encontrados nos perfis dos estudantes. De acordo com Rutherfoord [2006], um grupo formado com o critério da heterogeneidade tende a ser mais preferido por apresentar melhores resultados para o comportamento criativo. Em contra partida, Kulik e Kulik [1987] defendem que grupos homogêneos podem se sair melhores em tarefas específicas e, para formá-los, basta agrupar os alunos por similaridade de habilidades. Um grupo homogêneo pode ser caracterizado pela capacidade de agrupamento de estudantes com elevado grau de semelhança em relação a aspectos que influenciam a aprendizagem. 
Por outro lado, há propostas que consideram critérios de contextos gerais e específicos fundamentais para a formação de grupos [Hubscher, 2010]. Para isso, a abordagem de Hubscher combinou critérios gerais e específicos do contexto da aprendizagem, aplicados a algoritmos, para otimizar a atribuição dos membros. Embora tenha sugerido um novo tipo de grupo, chamado de Grupos Eventualmente Habilidosos, a aplicação da sua proposta não garante que a maioria das preferências seja atendida, pois as necessidades e características de cada turma podem variar.

\section{Metodologia}

O presente estudo está estruturado em duas etapas: um breve estudo de revisão acerca de formação de grupos, e; pesquisa com os tutores a distância do curso de Licenciatura em Computação na modalidade de Educação a Distância da Universidade Federal da Paraíba.

\subsection{Formação de grupos de aprendizagem: estudo de revisão sistemática}

Será utilizada a Revisão Sistemática na Literatura - RSL - com o objetivo de encontrar as práticas mais efetivas da área de estudo [Rumrill e Fitzgerald, 2001]. As etapas de RSL aqui descritas estão de acordo com Petersen et al., [2008]. O estudo pretende responder ao seguinte questionamento: em que medida as publicações na área tem contribuído com abordagens e estratégias, dentro do contexto internacional, em formação de grupos para a aprendizagem colaborativa?

\subsubsection{Definição dos conceitos-chave}

Os conceitos são identificados a partir da questão do estudo, a saber: a) Abordagem em grupo; b) Formação de grupos; c) Aprendizagem em grupo; d) Aprendizagem colaborativa; e) Atribuição de grupos; f) Formação de grupos de aprendizagem.

\subsubsection{Termos utilizados na busca (Strings)}

A construção da String de busca consiste na combinação dos conceitos-chave com operadores lógicos, como o "OR", que representa uma disjunção, e o "AND", representando uma conjunção. As Strings foram destacadas como: ("group approach" or "group strategy" or "group formation") AND ("group learning" or "group students" or "collaborative learning" or "learning groups").

\subsubsection{Portais de busca}

Os portais foram definidos conforme relevância na área, a saber: ACM Digital Library (http://dl.acm.org/); IEEE Xplore (http://ieeexplore.ieee.org/); Scopus (http://www.scopus.com/); ScienceDirect (http://www.sciencedirect.com/).

\subsubsection{Critérios de exclusão}

Os critérios de exclusão compreendem: estudos não escritos em inglês; artigos convidados, tutoriais, relatórios de workshop, teses, dissertações, relatórios técnicos, livros; documentos que não sejam artigos completos; artigos duplicados, que já foram encontrados em outras fontes; trabalhos sem os arquivos encontrados; estudos secundários, e; estudos que não correspondem à questão da pesquisa. 
CBIE-LACLO 2015

Anais dos Workshops do IV Congresso Brasileiro de Informática na Educação (CBIE 2015)

\subsection{Caracterização da pesquisa realizada com os tutores a distância}

Buscou-se explorar as preferências dos tutores a distância, as quais viabilizaram a especificação de perfis de grupos. A pesquisa foi descritiva pois permitiu a descrição da complexidade envolvida no fenômeno da aprendizagem em grupo [Mattar 2001].

\subsubsection{Universo e amostra}

O universo da população deste estudo correspondeu a 58 tutores do curso de Licenciatura em Computação na modalidade de Educação a Distância da Universidade Federal da Paraíba. A amostra foi composta por 12 tutores, o que corresponde 20,7\% do universo. A escolha da amostra dos participantes foi não probabilística por conveniência, tendo como único critério a disponibilidade para participação da pesquisa, conforme proposto em Mattar [2001]. Sob o julgamento dos pesquisadores, 20,7\% do universo da pesquisa que recebera o questionário iria, de fato, respondê-lo.

\subsubsection{Coleta de dados e locus da pesquisa}

A etapa de aplicação do questionário com os tutores teve por objetivo principal identificar quais as preferências que eles têm em relação às estratégias e critérios para agrupar estudantes, tais como o tamanho do grupo. Foi aplicado o questionário estruturado com questões fechadas e abertas. A aplicação dos questionários foi realizada entre 31 de outubro a 15 de novembro de 2014.

\subsubsection{Tratamento dos dados}

Após a aplicação dos questionários, os dados foram agrupados e tratados de forma qualitativa e quantitativa. Inicialmente procedeu-se a uma leitura geral dos dados coletados a fim de descobrir os núcleos de sentido para serem categorizados. A leitura flutuante forneceu uma compreensão generalizada do material coletado, e das respostas dos tutores emergiram duas grandes categorias: a possibilidade de configuração de grupos; e a definição dos Perfis.

\section{Resultados}

Os resultados apresentados nesta seção expõem a análise das publicações sobre formação de grupos de aprendizagem. Em seguida são identificados os perfis de grupos para apoiar a estratégia de formação.

Como resultados da execução das buscas, foi retornado um total de 625 publicações, sendo a maior quantidade encontrada no portal Science Direct, com 392 resultados, seguido de 123 trabalhos encontrados no portal Scopus, 104 publicações no portal ACM Digital Library e o portal IEEE Xplore Digital Library com 6 resultados.

\subsection{Seleção dos estudos primários}

Nesta etapa, realizou-se leitura e análise dos títulos e abstracts de todas as publicações do resultado da busca. Com isso, os trabalhos que correspondiam às questões de pesquisa foram armazenados em uma planilha para organização, indicando o portal encontrado, o título, o ano, o autor e o link de acesso ao arquivo. De posse de todos os trabalhos coletados, realizou-se a primeira filtragem, obtendo-se 124 trabalhos, os quais formam o panorama geral da distribuição das publicações, conforme Figura 1. 
CBIE-LACLO 2015

Anais dos Workshops do IV Congresso Brasileiro de Informática na Educação (CBIE 2015)

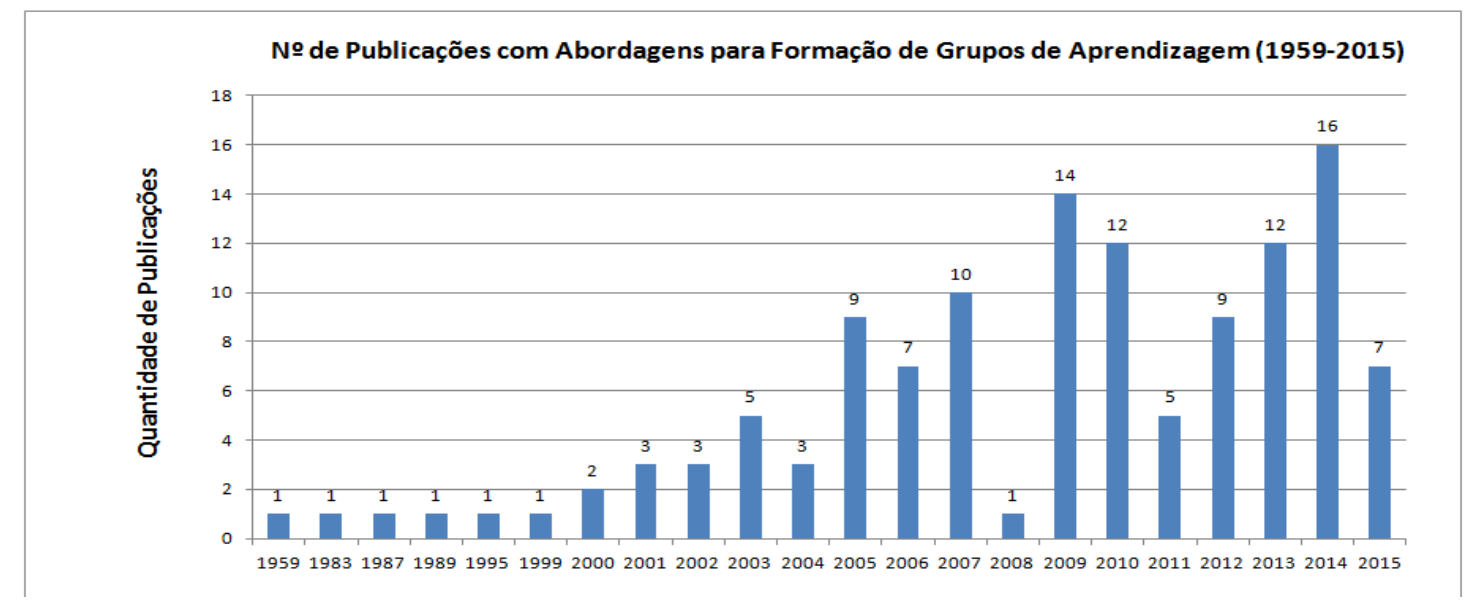

Figura 1. Gráfico da distribuição das publicações com abordagens para formação de grupos de aprendizagem, a partir da busca nos portais (1959-2015).

\subsection{Publicações que discutem estratégias e abordagens para a formação de grupos de aprendizagem}

A seleção das publicações relevantes foi realizada a partir da segunda filtragem dos trabalhos. Com a leitura da introdução e da conclusão de cada trabalho, obteve-se uma quantidade de 42 trabalhos, a partir desses foi realizada uma nova filtragem que teve como critério a seleção das publicações entre os anos de 2014 e 2015. Com isso, apenas quatro trabalhos desses realmente se comprometiam em propor abordagem para atribuição de estudantes em grupo, os quais foram selecionados e analisados quanto às abordagens propostas, às estratégias para a formação de grupos e seus resultados. Extraiu-se as informações relevantes dos trabalhos, dispondo-as no Quadro 1.

Quadro 1. Caracterização das estratégias encontradas em trabalhos recentes (2014-2015).

\begin{tabular}{|c|c|c|c|c|c|c|}
\hline & $\begin{array}{l}\text { Aspecto para a } \\
\text { Atribuição }\end{array}$ & $\begin{array}{c}\text { Estratégias } \\
\text { para obtenção } \\
\text { das } \\
\text { informações }\end{array}$ & $\begin{array}{l}\text { Tipos de } \\
\text { Grupos }\end{array}$ & $\begin{array}{l}\text { Método de } \\
\text { Validação }\end{array}$ & $\begin{array}{l}\text { Resultados } \\
\text { Pedagógicos }\end{array}$ & $\begin{array}{c}\text { Resultados } \\
\text { Computacionais }\end{array}$ \\
\hline $\begin{array}{l}\text { Sadegui } \\
\text { and } \\
\text { Kardan } \\
\text { [2015] }\end{array}$ & $\begin{array}{l}\text { Compatibilidade } \\
\text { para o trabalho } \\
\text { em grupo; } \\
\text { Dissimilaridade } \\
\text { entre os } \\
\text { membros. }\end{array}$ & $\begin{array}{l}\text { Modelagem dos } \\
\text { alunos pelas } \\
\text { informações no } \\
\text { sistema de } \\
\text { gerenciamento } \\
\text { de curso. }\end{array}$ & $\begin{array}{l}\text { Grupos } \\
\text { Aleatórios; } \\
\text { Homogêneos, } \\
\text { e; } \\
\text { Heterogêneos. }\end{array}$ & $\begin{array}{l}\text { Tempo de } \\
\text { execução do } \\
\text { algoritmo; } \\
\text { Satisfação e } \\
\text { conhecimentos } \\
\text { adquiridos. } \\
\end{array}$ & $\begin{array}{l}\text { Maior satisfação } \\
\text { e novos } \\
\text { conhecimentos } \\
\text { adquiridos pelos } \\
\text { alunos. }\end{array}$ & $\begin{array}{l}\text { Facilidade para } \\
\text { ajustes de } \\
\text { parâmetros; Tempo } \\
\text { satisfatório na } \\
\text { formação dos } \\
\text { grupos. }\end{array}$ \\
\hline $\begin{array}{l}\text { Kardan } \\
\text { and } \\
\text { Sadegui } \\
{[2014]}\end{array}$ & $\begin{array}{l}\text { Similaridade de } \\
\text { habilidades, com } \\
\text { fórmula } \\
\text { matemática } \\
\text { aplicada ao } \\
\text { algoritmo } K \text { - } \\
\text { means. }\end{array}$ & $\begin{array}{l}\text { Extração por } \\
\text { meio de } \\
\text { palavras-chaves } \\
\text { dos perfis dos } \\
\text { alunos no } \\
\text { sistema de } \\
\text { gerenciamento } \\
\text { de cursos. }\end{array}$ & $\begin{array}{l}\text { Grupo } \\
\text { Homogêneo; }\end{array}$ & $\begin{array}{l}\text { Nível de } \\
\text { satisfação dos } \\
\text { alunos; } \\
\text { Acurácia do } \\
\text { algoritmo. }\end{array}$ & $\begin{array}{l}\text { Grupos com } \\
\text { elevado grau de } \\
\text { semelhança } \\
\text { entre seus } \\
\text { membros } \\
\text { garantiu bom } \\
\text { nível de } \\
\text { satisfação. }\end{array}$ & $\begin{array}{l}\text { Tempo de } \\
\text { execução do } \\
\text { algoritmo como } \\
\text { inferior a } 1 \mathrm{~s} \text {. } \\
\text { Desempenho do } \\
\text { algoritmo diminui } \\
\text { quando se aumenta } \\
\text { o número de } \\
\text { estudantes. }\end{array}$ \\
\hline $\begin{array}{l}\text { Jagadish } \\
\text { [2014] }\end{array}$ & $\begin{array}{l}\text { Similaridade de } \\
\text { conhecimentos. }\end{array}$ & $\begin{array}{l}\text { Auto indicação } \\
\text { do nível de } \\
\text { conhecimento } \\
\text { em relação à } \\
\text { diferentes } \\
\text { habilidades. }\end{array}$ & $\begin{array}{l}\text { Grupo } \\
\text { Homogêneo; }\end{array}$ & $\begin{array}{l}\text { Comparação do } \\
\text { desempenho dos } \\
\text { alunos } \\
\text { trabalhando } \\
\text { individualmente } \\
\text { e em grupo. }\end{array}$ & $\begin{array}{l}\text { Melhorias no } \\
\text { desempenho do } \\
\text { aprendizado dos } \\
\text { alunos em grupo } \\
\text { em relação ao } \\
\text { aprendizado } \\
\text { individual. }\end{array}$ & $\begin{array}{l}\text { Uso do algoritmo } \\
\text { K-NN eliminou a } \\
\text { forma manual de } \\
\text { atribuição e } \\
\text { equilibrou o nível } \\
\text { de conhecimento } \\
\text { entre os membros. }\end{array}$ \\
\hline
\end{tabular}




\section{CBIE-LACLO 2015}

Anais dos Workshops do IV Congresso Brasileiro de Informática na Educação (CBIE 2015)

\begin{tabular}{|l|l|l|l|l|l|l|}
\hline $\begin{array}{l}\text { Zheng } \\
\text { and } \\
\text { Pinkwart } \\
{[2014]}\end{array}$ & $\begin{array}{l}\text { Distribuição } \\
\text { normalizada de } \\
\text { gênero e } \\
\text { diversidade. }\end{array}$ & $\begin{array}{l}\text { Sistema de } \\
\text { gerenciamento } \\
\text { de curso. }\end{array}$ & Heterogêneo & $\begin{array}{l}\text { Comparação do } \\
\text { tempo entre os } \\
\text { métodos DPSO, } \\
\text { exaustivo e } \\
\text { aleatório. }\end{array}$ & $\begin{array}{l}\text { Aspectos de } \\
\text { avaliação } \\
\text { pedagógica não } \\
\text { foram } \\
\text { encontrados. }\end{array}$ & $\begin{array}{l}\text { O método DPSO } \\
\text { teve custo de } \\
\text { tempo menor que o } \\
\text { método exaustivo. } \\
\text { O método aleatório } \\
\text { foi o melhor entre } \\
\text { os três. }\end{array}$ \\
\hline
\end{tabular}

Analisando o Quadro 1, constatou-se que os trabalhos recentes, que propõem abordagens para formação de grupos, consideram as habilidades dos estudantes como fatores determinantes nesse processo. Além disso, foi percebido o interesse na descoberta de conhecimento em base de dados como forma de obtenção das informações relevantes dos estudantes [Zheng e Pinkwart, 2014], [Kardan e Sadegui, 2014], [Sadegui e Kardan, 2015], sendo que estes dois últimos consideram o nível de satisfação dos estudantes como forma de validar as estratégias de atribuição e mensurar os efeitos do trabalho em grupo. Entretanto, fica evidente que as abordagens apresentadas pouco exploram a diversidade de tipos de grupos, conforme proposto em Muehlenbrock [2006] e Hubscher [2010], conservando os seus experimentos apenas em torno dos grupos formados com critérios de heterogeneidade, homogeneidade ou aleatoriedade. Verificou-se ainda maior ênfase dada aos resultados computacionais, principalmente o desempenho do algoritmo de otimização utilizado, exceto em Jagadish [2014]. Isso é importante notar, pois, os resultados pedagógicos parecem, a priori, produzir reflexos positivos pouco conclusivos acerca do trabalho colaborativo.

\subsection{Preferências dos tutores}

As respostas da pesquisa com os tutores possibilitaram verificar que a maioria $(83,33 \%)$ afirma ser viável criar grupos colaborativos na EaD. A maioria dos tutores considera que o motivo para o interesse em criar grupos consiste na busca pelo aumento da interação entre os alunos (83,33\%). Quanto à forma de agrupamento, os resultados da pesquisa sugerem grupos por disponibilidade de horário $(41,66 \%)$, grupos por polo $(41,66 \%)$, grupos por participação na plataforma da $\mathrm{EaD}(33,33 \%)$ e grupos complementares (25\%). Do ponto de vista do tamanho e da presença ou não de líder no grupo, os tutores afirmam que o tamanho ideal para os grupos é de quatro membros por grupo $(33,33 \%)$ e apenas $8,33 \%$ indicou grupos com líder. Quanto à duração dos grupos, a maioria acredita ser mais viável utilizar os mesmos grupos durante um semestre acadêmico $(41,66 \%)$.

\subsection{Especificação dos Perfis de grupos}

Com base nos resultados e no estudo da literatura [Oakley, 2004], [Rutherfoord, 2006], [Kulik e Kulik, 1987], [Muehlenbrock, 2006], [Hubscher, 2010], este trabalho propõe a especificação dos seguintes Perfis de grupos, conforme Tabela 1: 
CBIE-LACLO 2015

Anais dos Workshops do IV Congresso Brasileiro de Informática na Educação (CBIE 2015)

Tabela 1. Especificação de Perfis de Grupos.

\begin{tabular}{|c|c|c|}
\hline Perfil & Nome & Descrição \\
\hline 1 & Grupo por Disponibilidade & $\begin{array}{l}\text { Membros selecionados quanto à compatibilidade de } \\
\text { horário. }\end{array}$ \\
\hline 2 & Grupo por Polo & Membros selecionados por Polo de apoio à EaD. \\
\hline 3 & Grupo por Participação & $\begin{array}{c}\text { Membros selecionados por quantidade de } \\
\text { participação nas atividades propostas na plataforma } \\
\text { Moodle. }\end{array}$ \\
\hline 4 & $\begin{array}{l}\text { Grupo por habilidades } \\
\text { heterogêneas }\end{array}$ & $\begin{array}{l}\text { Membros selecionados de acordo com as } \\
\text { habilidades. }\end{array}$ \\
\hline 5 & Grupo Complementar & $\begin{array}{l}\text { Membros selecionados por habilidades } \\
\text { complementares. }\end{array}$ \\
\hline
\end{tabular}

Analisando a Tabela 1, que descreve o conjunto de perfis de grupo baseados nas preferências de tutores, verifica-se que há uma ampla diversidade de formas de agrupamento de estudantes a ser explorada. Uma proposta de abordagem para formar grupos com base no contexto do estudante é aplicar técnicas de mineração de dados educacionais [Zheng e Pinkwart, 2014], [Kardan e Sadegui, 2014]. O conhecimento sobre a frequência de horários de acesso a uma plataforma de curso a distância ou as informações sobre a participação em fóruns de discussão, por exemplo, podem caracterizar informações determinantes para formar grupos com estudantes que têm compatibilidade de horário de estudo ou grupos que combinam alunos mais participantes com alunos mais tímidos, oportunizando o estímulo mútuo dentro do grupo, refletindo a utilidade dessas informações para melhorar os processo de aprendizagem na EaD [Sadegui e Kardan, 2015].

\subsubsection{Uma aplicação de modelagem matemática para o perfil de grupo por habilidades heterogêneas}

Os perfis de grupos podem ser modelados matematicamente [Kardan e Sadeghi, 2014], visando a sua utilização por um algoritmo de agrupamento [Facelli et al., 2011]. O grupo por habilidades heterogêneas pode ser obtido buscando maximizar a distância entre as habilidades dos estudantes [Hubscher, 2010]. A instanciação desse algoritmo busca minimizar o erro quadrático, como critério de agrupamento, para maximizar a variação entre clusters. O erro quadrático é dado pela equação apresentada na Figura 2, onde $d\left(h i, \bar{h}^{(\mathrm{j})}\right)$ é a distância euclidiana do valor da habilidade $h$ de um dado objeto $i$ e o centróide $\bar{h}^{(\mathrm{j})}$, utilizada como medida de dissimilaridade.

$$
E=\sum_{j=1}^{k} \sum_{h i \in C j} d\left(h i, \bar{h}^{(j)}\right)
$$

Figura 2. Fórmula do Erro Quadrático [Facelli et al., 2011].

Grupos heterogêneos podem ser obtidos com a aplicação do algoritmo k-médias, o qual agrupa os membros no número pré-definido de $\mathrm{k}$ grupos [Facelli et al., 2011]. Por exemplo, considerando os valores apresentados por quatro estudantes A, B, C e D entre os níveis de 0 a 5 nas habilidades de português e de matemática, com os quais pretende-se formar dois grupos heterogêneos, conforme Tabela 2: 
Tabela 2: Valores dos níveis de habilidades entre estudantes.

\begin{tabular}{c|c|c|c|c}
\hline \multirow{2}{*}{ Habilidade } & \multicolumn{5}{|c}{ Aluno } \\
\cline { 2 - 5 } & A & B & C & D \\
\hline Português & 5 & 4 & 1 & 0 \\
\hline Matemática & 0 & 1 & 4 & 5 \\
\hline
\end{tabular}

A aplicação do algoritmo utilizando esses valores permitirá, dentre todas as possibilidades de combinação, formar pares de alunos com níveis de habilidades mais distintos entre si. Estudantes com habilidades distintas entre si podem apresentar melhor desempenho para a criatividade, o que pode potencializar a aprendizagem em grupo [Rutherfoord, 2006]. A partir desse exemplo, os demais perfis de grupos podem ser representados através da similaridade ou dissimilaridade entre os estudantes, buscando maximizar ou minimizar a distância entre as suas características.

\section{Conclusão}

Diferentemente dos tipos de grupos encontrados na literatura, propostos a atribuir estudantes para o trabalho colaborativo, os perfis de grupos especificados neste trabalho viabilizam uma maior abrangência das preferências e dos critérios para a formação de grupos de aprendizagem, tendo em vista serem fundamentadas nas experiências de professores mediadores da $\mathrm{EaD}$.

Dentre os aspectos significativos desse estudo destaca-se a revisão na literatura, que possibilitou a compreensão de que as publicações na área têm focado, em geral, nas diferentes estratégias para a otimização de algoritmos de agrupamento. Entretanto, do ponto de vista pedagógico, as publicações encontradas pouco dão atenção às preferências dos tutores que mediam a educação nessa modalidade. A realização da pesquisa mostrou que os tutores possuem informações decisivas à formação diferentes tipos de grupos, o que parece ainda ser desconhecido pela literatura.

Com base nos fundamentos teóricos norteadores desse estudo, verificou-se a possibilidade de extrair informações de estudantes armazenadas no banco de dados do Ambiente Virtual de Aprendizagem de apoio ao curso em questão, aliando-as aos perfis especificados, para criar grupos significativos. Diante disso, pretende-se como trabalhos futuros realizar a modelagem matemática dos grupos e, a partir do uso de técnicas de Aprendizado de Máquina, apoiar a tarefa de formação de grupos de aprendizagem na EAD com uma turma de estudantes do curso de Licenciatura em Computação à distância da Universidade Federal da Paraíba. Com isso, busca-se avaliar até que ponto os grupos especificados contribuem para o desempenho acadêmico dos estudantes. Em aplicações futuras, espera-se que os tutores virtuais possam concentrar seus esforços na condução das atividades e no acompanhamento da aprendizagem na EaD.

\section{Referências}

Brito J. A., Melo Filho I. J., Carvalho R. S., Melo R. M., Gomes A. S., (2011), Interfaces colaborativas para atividades assíncronas em fórum de discussão. In Anais do XXII SBIE - XVII WIE - Aracajú - SE.

Chapman, K.J., Meuter, M., Toy, D., Wright, L. (2006). Can't We Pick Our Own Groups? The Influence of Group Selection Method on Group Dynamics and Outcomes, J. Management Education, vol. 30, no. 4, pp. 557-569. 


\section{CBIE-LACLO 2015}

Anais dos Workshops do IV Congresso Brasileiro de Informática na Educação (CBIE 2015)

Dillenbourg, P. (1999). What do you mean by collaborative learning? In: Dillenbourg P. (eds): Collaborativelearning: Cognitive and Computational Approaches. Oxford: Elsevier 1-19.

Faceli, K. ; lorena, A. C. ; Gama, J. M. P. ; Carvalho, A. C. P. L. F. . Inteligência Artificial - uma abordagem de Aprendizado de Máquina. Rio de Janeiro: LTC, 2011.

Hubscher, R. (2010). Assigning students to groups using general and context specific criteria. IEEE Transactions on Learning Technologies, 3(3), 178-189.

Jagadish,D. (2014). Grouping in collaborative e-learning environment based on interaction among students. In International Conference on Recent Trends in Information Technology.

Kardan, A.; Sadeghi, H., (2014), Modeling the learner group formation problem in computer-supported collaborative learning using mathematical programming. The 8th National and 5th International Conference on e-Learning and e-Teaching, Tehran, Iran, 2014.

Kulik, J.A., Kulik, C.L.C., (1987). Effects of Ability Grouping on Student Achievement, Equity and Excellence, vol. 23, pp. 22-30.

Lipponen, L. (2002). Exploring Foundations for Computer Supported Collaborative Learning. Proceedings of CSCL 2002, pp. 72-81.

Mahenthiran, S., Rouse, P.J. (2000). The Impact of Group Selection on Student Performance and Satisfaction," Int'1 J. Educational Management, vol. 14, no. 6, pp. 255-265.

Mattar, N. F. (2001). Pesquisa de Marketing. São Paulo: 3. ed. Atlas.

Muehlenbrock, M. (2006). Learning Group Formation Based on Learner Profile and Context, Int'1 J. E-Learning, vol. 5, no. 1, pp. 19-24.

Oakley, B., Felder, R.M., Brent, R., Elhajj, I. (2004). Turning Student Groups into Effective Teams, J. Student Centered Learning, vol. 2, no. 1, pp. 8-33.

Palincsar, A.S., Brown, A.L. (1984). Reciprocal Teaching of Comprehension-Fostering and Comprehension-Monitoring Activities, Cognition and Instruction, vol. 1, no. 2, pp. 117-175.

Petersen, K., Feldt, R., Mujtaba, S., and Mattsson, M. (2008). Systematic mapping studies in software engineering. In International Conference on Evaluation and Assessment in Software Engineering.

Prado, M. E. B. B., (2006), A Mediação Pedagógica: suas relações e interdependências. In Anais do XVII Simpósio Brasileiro de Informática na Educação - SBIE - Brasília, DF.

Ramos, J. L. C., Rodrigues, R. L., Silva, J. C. S., Gomes, A. S., (2014). Analisando Fatores que Afetam o Desempenho de Estudantes Iniciantes em um Curso a Distância. In Anais do XXV Simpósio Brasileiro de Informática na Educação (SBIE 2014 - Dourados-MS).

Roschelle, J. and Teasley, S. (1995). The construction of shared knowledge in collaborative problem solving. In O'Malley, C.E., (ed.), Computer Supported Collaborative Learning. pages 69--97. Springer-Verlag, Heidelberg. 
Rosenshine B., Meister, C. (1994). Reciprocal Teaching: A Review of the Research, Rev. of Educational Research, vol. 64, pp. 479-530.

Rumrill, P. D., \& Fitzgerald, S. M. (2001). Speaking of research: Using narrative literature reviews to build a scientific knowledge base. Work, 16, 165-170.

Rutherfoord, R.H. (2006). Using Personality Inventories to Form Teams for Class Projects: A Case Study, Proc. Seventh Conf. Information Technology Education (SIGITE '06), pp. 9-14.

Sadeghi, H.; Kardan, A., (2015), A novel justice-based linear model for optimal learner group formation in computer-supported collaborative learning environments. Computers in Human Behavior, 48, 2015, 436-447.

Santos L. N., Castro Jr. A. N., Castro T. H. C., (2007). Alteração no Modelo de Grupos do Moodle para Apoiar a Colaboração. In Anais do XVIII Simpósio Brasileiro de Informática na Educação - SBIE - São Paulo - SP.

Zheng, Z.; Pinkwart, N. (2014). A Discrete Particle Swarm Optimization Approach to Compose Heterogeneous Learning Groups. In IEEE 14th International Conference on Advanced Learning Technologies. 